\title{
Dengue hemorrhagic fever with multiple abscesses: An unusual presentation
}

\author{
Kunal Bhatia, Manisha Thakur, YC Porwal, UC Kansra, Arun Iyer
}

\begin{abstract}
Introduction: Dengue is a common arboviral illness endemic in India. Concurrent bacterial co-infection in dengue is rare and often underreported. Case Report: We report a case of an 18-year-old male with dengue hemorrhagic fever presenting as multiple abscesses due to a concomitant bacterial co-infection. Surgical intervention was done and the pus aspirated isolated Escherichia coli, although blood cultures were negative. The patient responded favorably after starting an antimicrobial regimen based on pus culture sensitivity reports and was discharged thereafter. Conclusion: Clinicians should be aware of the possibility of a concurrent bacterial co-infection in patients with dengue virus infection/dengue hemorrhagic fever so as to initiate a timely additional antimicrobial treatment.
\end{abstract}

Kunal Bhatia ${ }^{1}$, Manisha Thakur ${ }^{2}$, YC Porwal ${ }^{3}$, UC Kansra ${ }^{4}$, Arun lyer ${ }^{5}$

Affiliations: ${ }^{1}$ MBBS, PGY-II (MD Medicine), Department of Medicine, Vardhman Mahavir Medical College and Safdarjung Hosiptal, New Delhi, India; ${ }^{2} \mathrm{MD}$ Medicine, Associate Professor, Department of Medicine, Vardhman Mahavir Medical College and Safdarjung Hospital, New Delhi, India; ${ }^{3} \mathrm{MD}$ Medicine, Associate Professor, Department of Medicine, Vardhman Mahavir Medical College and Safdarjung Hospital, New Delhi, India; ${ }^{4} \mathrm{MD}$ Medicine, FICP, FIACM, Professor, Department of Medicine, Vardhman Mahavir Medical College and Safdarjung Hospital, New Delhi, India; ${ }^{5}$ MBBS, PGY-III (MD Medicine), Department of Medicine,Vardhman Mahavir Medical College and Safdarjung Hospital, New Delhi, India.

Corresponding Author: Dr. Kunal Bhatia, B-482, Ground Floor, Sushant Lok I, Gurgaon, Haryana, India Postal Code: 122002; Phone: 91-965-0927471; Email: kunalbhatia.del@gmail.com

Received: 24 September 2012

Accepted: 17 November 2012

Published: 01 June 2013
Keywords: Dengue fever, Dengue hemorrhagic Fever, Unusual presentation, Abscesses

$* * * * * * * * *$

Bhatia K, Thakur M, Porwal YC, Kansra UC, Iyer A. Dengue hemorrhagic fever with multiple abscesses: An unusual presentation. International Journal of Case Reports and Images 2013;4(6):326-329.

$* * * * * * * * *$

doi:10.5348/ijcri-2013-06-323-CR-8

\section{INTRODUCTION}

Dengue is the most rapidly spreading mosquitoborne viral disease in the world with an estimated 50 million dengue infections occurring annually and approximately 2.5 billion people live in dengue endemic countries [1]. The clinical spectrum of dengue is highly variable, ranging from a mild flu-like syndrome to severe disease, with shock and hemorrhage. However, in the recent years various authors around the globe have reported atypical manifestations associated with dengue fever. Some unusual manifestations of dengue fever that have been previously described include fulminating liver failure, rhabdomyolysis, acute renal failure, myocarditis, neurological disturbances of encephalopathy, Gullian-Barré syndrome and transverse myelitis [2-6]. Here we are reporting a case of dengue hemorrhagic fever associated with multiple abscesses.

\section{CASE REPORT}

A 18-year-old male, mason by profession, presented with high grade fever with chills, myalgia, joint pains and prostration for two days followed by pain over the right shoulder, right anterior chest wall and the left 
gluteal region. On the 3rd day of fever, patient started complaining of painful swellings over the above mentioned areas along with black tarry loose stools (2-3 episodes/day) with abdominal distension which was gradual in onset and progressive. The patient got admitted to a private hospital in Agra where he was found to be dengue NS1Ag positive. He also underwent needle aspiration of the swelling present over the right shoulder and was given broad spectrum antibiotics (intravenous amoxicillin-clavulanic acid, azithromycin and teicoplanin) and supportive care for three days. However, the patient's general condition did not improve and he presented to us in the emergency room with a diagnosis of dengue hemorrhagic fever complicated by multiple abscesses. Further history taking revealed that the patient continued to have fever for the past seven days along with passage of black tarry loose stools (2-3 episodes/day), abdominal distension, painful swellings involving the right shoulder, right anterior chest wall and left gluteal region for the last five days. No history of petechial rashes or bleeding from any other site could be elicited. History of trauma, intramuscular injections, intravenous drug abuse, and diabetes mellitus were negative.

On physical examination, patient was pale, febrile with a temperature of $102^{\circ} \mathrm{F}$, pulse rate of $98 / \mathrm{min}$, blood pressure 124/60 $\mathrm{mmHg}$ and Hess test/tourniquet test was negative. On local examination, a swelling $1.5 \times 1 \mathrm{~cm}$ over the right shoulder, a $4 \times 3 \mathrm{~cm}$ swelling over the left gluteal region and another swelling of $3 \times 3 \mathrm{~cm}$ over the right anterior chest wall were seen, which were hot, tender, firm with diffuse margins, showing fluctuation and surrounding induration; suggestive of abscesses. Respiratory, cardiovascular and neurological examination were within normal limits. Per abdomen examination revealed distended abdomen with presence of free fluid and no organomegaly. Laboratory investigations (on the day of admission) revealed hemoglobin of $7.2 \mathrm{~g} / \mathrm{dL}$ with peripheral smear showing normocytic normochromic anemia and no malarial parasite, total leucocyte count of $7400 / \mathrm{mm}^{3}$ (neutrophils 65\%, lymphocytes $31 \%$, monocytes $2 \%$, eosinophils 2\%), platelet count $1.9 \times 10^{4} / \mathrm{mm}^{3}$, PCV 31.6\%, serum bilirubin $1.1 \mathrm{mg} / \mathrm{dL}$, aspartate transaminase $153 \mathrm{IU} / \mathrm{L}$, alanine transaminase $65 \mathrm{IU} / \mathrm{L}$, alkaline phosphatase $267 \mathrm{IU} / \mathrm{L}$. Renal functions were within normal limits. Chest roentgenography showed minimal bilateral pleural effusion. Ultrasonography of abdomen revealed partially distended gallbladder with edematous and thickened wall, mild hepatosplenomegaly and moderate ascites suggestive of polyserositis. Samples for blood culture and urine culture were withdrawn and patient was started on broad spectrum intravenous antibiotics (ceftazidime + vancomycin).

Ultrasonography of left gluteal region showed a lobulated complex cystic lesion with internal echoes with approximate size of $3.9 \times 1.9 \times 3.3 \mathrm{~cm}\left(13.5 \mathrm{~cm}^{3}\right)$ located in the intramuscular plain with adjacent soft tissue edema (Figure 1). Another abscess with approximate size of $3.54 \times 1.52 \times 2.1 \mathrm{~cm}$ was located on the right anterior chest wall with similar features (Figure 2).

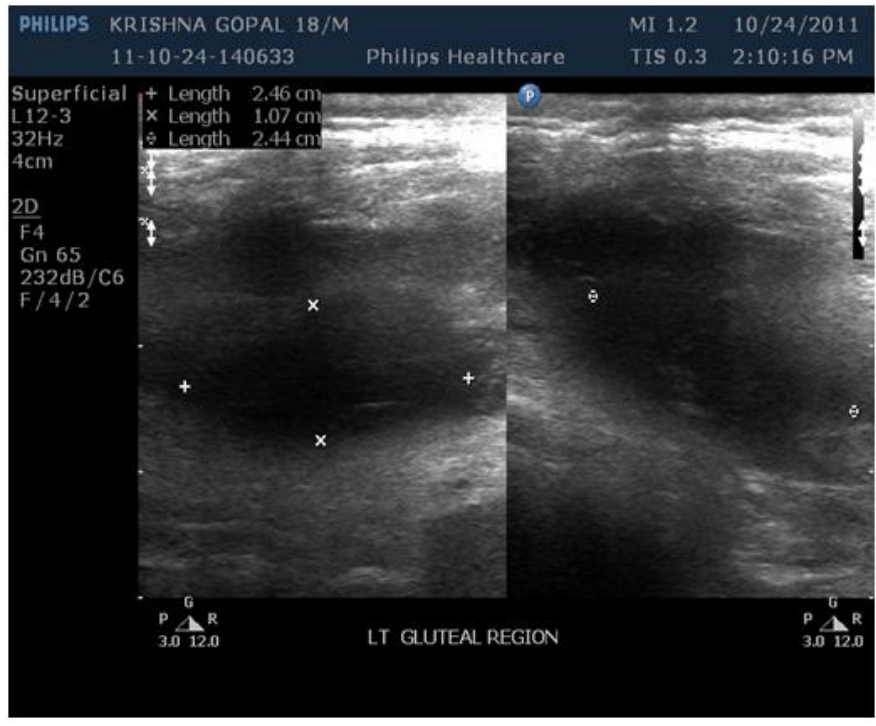

Figure 1: Showing an abscess located at the left gluteal region.

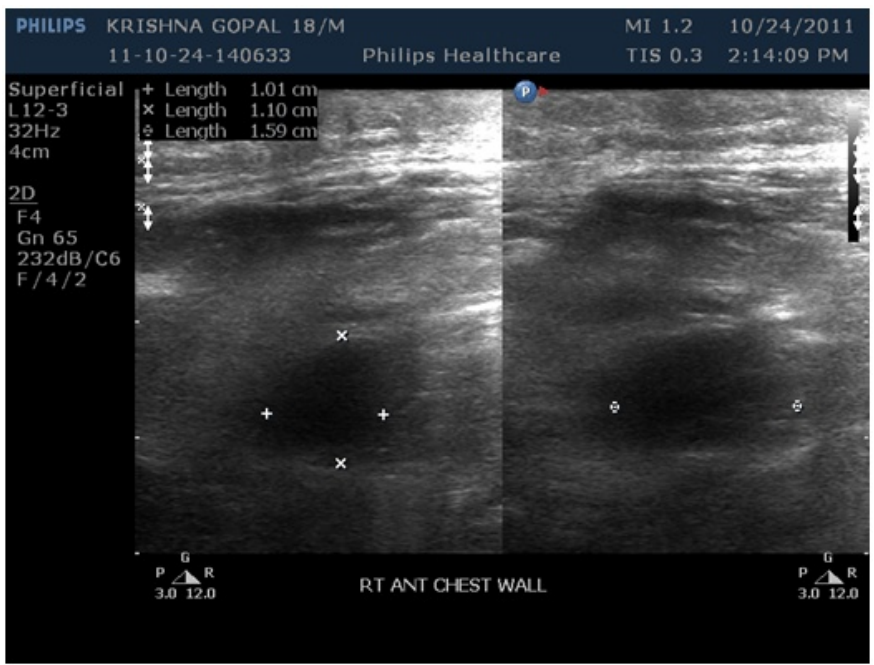

Figure 2: Showing an abscess on the right anterior chest wall.

On the 9th day of the illness, incision and drainage of the abscesses was done after transfusing platelet rich plasma; and the pus aspirated was sent for microbiological examination, culture and sensitivity. No organisms were identified in the blood and urine cultures. Dengue serology was positive for IgM antibodies (MAC-ELISA) and HIV-ELISA was nonreactive. Microbiological investigations showed gram negative bacilli with a background of inflammatory cells and necrotic debris. Culture and sensitivity of the aspirate from all the three sites showed a growth of Escherichia coli which was sensitive to ertapenem and meropenem.

The patient continued to have fever, malena and generalized weakness with hemoglobin dropping to 6.5 $\mathrm{g} / \mathrm{dL}$ with a PCV of $24.7 \%$ and platelet count decreased to $10,000 / \mathrm{mm}^{3}$ on day 10 . Therefore, in accordance with the 2009 WHO guidelines for management of dengue 
hemorrhagic fever, patient was transfused with 2 units of fresh whole blood and 4 units of platelet rich plasma. Following blood transfusion the patients' hemoglobin increased to $7.9 \mathrm{~g} / \mathrm{dL}$ with platelet count of $1.9 \times 10^{4} / \mathrm{mm}^{3}$ and a PCV of $29.1 \%$; but fever persisted. Subsequently, the patient was shifted onto intravenous meropenem based on pus culture sensitivity report. Antimicrobial therapy with antiseptic dressing of the drainage sites was continued for the next few days along with platelet count and hematocrit monitoring daily.

On the 14th day of the illness, patient started showing signs of improvement with platelet count increasing to $3.9 \times 10^{4} / \mathrm{mm}^{3}$ and a PCV of $37.5 \%$. Patient became afebrile with no signs of bleeding from any site and had persistently increasing platelet counts thereafter. Hence, the patient was discharged after completing 7 days of antimicrobial therapy.

\section{DISCUSSION}

Dengue infection is an arboviral disease endemic in many parts of the world including India. Dengue infections are usually asymptomatic, but can present with classic dengue fever, dengue hemorrhagic fever or dengue shock syndrome. In the last few years there has been an increased spread of the dengue fever disease worldwide which has allowed the observation of a large number of patients with atypical clinical presentations [2-6].

The occurrence of bacterial co-infection in patients with dengue has been noted by some authors, but the available information comes from anecdotic reports. The microorganisms that have been identified to occur simultaneously with dengue virus infection include Gram-negative Enterobacteriaceae species, Enterococcus faecalis, Mycobacterium tuberculosis, Klebsiella pneumonia, Klebisella ozaenae, Moraxella lacunata and Staphylococcus aureus [7, 8].

Our patient had dengue hemorrhagic fever complicated by formation of multiple abscesses involving different regions of the body. The isolation of Escherichia coli in the pus aspirates can be explained by the disintegration of intestinal mucosal barrier, possibly through endothelial damage or intestinal hemorrhage, resulting in creation of a portal of entry for pathogens that normally inhabit the intestinal tract to enter the circulation [9]. However, isolation of E. coli in the pus with blood and urine cultures being negative can be explained by the use of broad-spectrum antibiotics empirically before taking blood and urine samples for culture. The occurrence of bacterial co-infection in our patient can also be explained with the state of immunosuppression caused by the virus, as acute dengue infection modulates the immune system by causing impaired $\mathrm{T}$ cell proliferation and cell mediated responses leading to immunosuppression and increased risk of acquiring other complicating infections [10].

The possibility of co-infection was considered in our patient, as the fever persisted beyond the anticipated period characteristic of dengue fever along with the presence of multiple soft tissue abscesses. Hence, it is important for clinicians to recognize dual infection in patients presenting with dengue and non-resolving fever as concurrent bacteremia can be easily overlooked especially in dengue endemic areas.

\section{CONCLUSION}

Dengue fever with a concurrent bacterial infection is rare and often underreported. As the spread of dengue virus increases, clinicians need to be vigilant about the unusual manifestations of the disease and the importance of recognizing them early. The possibility of a concomitant bacterial infection in a patient with dengue virus infection should be taken into consideration when the fever lasts for more than $5^{-7}$ days so as to initiate timely appropriate antimicrobial therapy.

$$
* * * * * * * * *
$$

\section{Author Contributions}

Kunal Bhatia - Conception and design, Acquisition of data, Analysis and interpretation of data, Drafting the article, Critical revision of the article, Final approval of the version to be published

Manisha Thakur - Conception and design, Acquisition of data, Analysis and interpretation of data, Drafting the article, Critical revision of the article, Final approval of the version to be published

YC Porwal - Conception and design, Acquisition of data, Analysis and interpretation of data, Drafting the article, Critical revision of the article, Final approval of the version to be published

UC Kansra - Conception and design, Acquisition of data, Analysis and interpretation of data, Drafting the article, Critical revision of the article, Final approval of the version to be published

Arun Iyer - Conception and design, Acquisition of data, Analysis and interpretation of data, Drafting the article, Critical revision of the article, Final approval of the version to be published

\section{Guarantor}

The corresponding author is the guarantor of submission.

\section{Conflict of Interest}

Authors declare no conflict of interest.

\section{Copyright}

(C) Kunal Bhatia et al. 2013; This article is distributed under the terms of Creative Commons Attribution 3.0 License which permits unrestricted use, distribution and reproduction in any means provided the original authors and original publisher are properly credited. (Please see www.ijcasereportsandimages.com /copyright-policy.php for more information.) 


\section{REFERENCES}

1. World Health Organization. Dengue: guidelines for diagnosis, treatment, prevention and control -- New edition. Geneva 2009.

2. Jhamb R, Kashyap B, Ranga GS, Kumar A. Dengue fever presenting as acute liver failure--a case report. Asian Pac J Trop Med 2011 Apr;4(4):323-4.

3. Lee IK, Liu JW, Yang KD. Clinical characteristics, risk factors, and outcomes in adults experiencing dengue hemorrhagic fever complicated with acute renal failure. Am J Trop Med Hyg 2009 Apr;80(4):651-5.

4. Lee IK, Lee WH, Liu JW, Yang KD. Acute myocarditis in dengue hemorrhagic fever: a case report and review of cardiac complications in dengue-affected patients. Int $J$ Infect Dis 2010 Oct;14(10):e919-22.

5. Soares CN, Faria LC, Peralta JM, de Freitas MR, Puccioni-Sohler M. Dengue infection: neurological manifestations and cerebrospinal fluid (CSF) analysis. J Neurol Sci 2006 Nov 1;249(1):19-24.
6. Seet RC, Lim EC, Wilder-Smith EP. Acute transverse myelitis following dengue virus infection. J Clin Virol 2006 Mar;35(3):310-2.

7. Lee IK, Liu JW, Yang KD. Clinical characteristics and risk factors for concurrent bacteremia in adults with dengue hemorrhage fever. Am J Trop Med Hyg 2005;72(2):221-6.

8. Chai LY, Lim PL, Lee CC, et al. Cluster of Staphylococcus aureus and dengue co-infection in Singapore. Ann Acad Med Singapore 2007;36(10):847-50.

9. Lin CF, Lei HY, Shiau AL, et al. Endothelial cell apoptosis induced by antibodies against dengue virus nonstructural protein 1 via production of nitric oxide. J Immunol 2002;169(2):657-64.

10. Mathew A, Kurane I, Green S, et al. Impaired T cell proliferation in acute dengue infection. J Immunol 1999 May 1;162(9):5609-15.
Access full text article on other devices

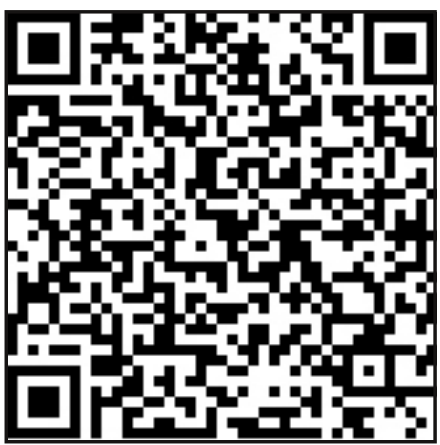

Access PDF of article on other devices

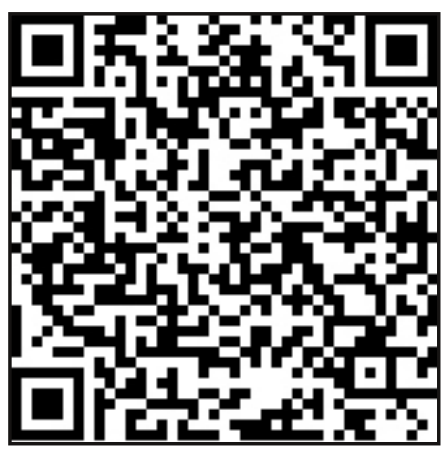

